\title{
Robust Kalman Filtering for Uncertain Discrete Time-Varying System with State-Delay
}

\author{
Jun-Hui Zheng ${ }^{1, \mathrm{a}}$ and Quan Gan ${ }^{1, \mathrm{~b}}$ \\ ${ }^{1}$ College of Computer Science and Technology, Pingdingshan University, \\ Pingdingshan Henan, China 467000 \\ apdszhengjunhui@163.com, ${ }^{b}$ spring.gump@163.com
}

\begin{abstract}
In this paper, a robust finite-horizon kalman filter for discrete time-varying uncertain system with constant state-delay both in state and output matrices. The system parameters uncertainties are expected in the state, output and white noise covariance matrices. The upper bound on the estimation error covariance is given and minimized. In addition, the filter parameters state, state delay and gain matrices are optimized to give a minimal upper bound on the estimation error covariance for all admissible uncertainties. A numerical example is presented to illustrate the applicability of this method.
\end{abstract}

Keywords: Uncertain Discrete time-varying systems, State Delay, Kalman filtering, Robust filtering

\section{Introduction}

The idea of kalman filter is to design a filter to minimize the estimation error covariance. It is successfully used to solve estimation problems of state variables for state real-time dynamic systems when the available observations are noisy. However, the optimality and guaranteed stability margin of kalman filter holds only when the system model parameters and the statistics of the noises are exactly known [1]. When the system suffers from uncertainties: uncertainty in modeling parameters and uncertainty in statistics of the noises, the performance of the standard kalman filtering could be greatly degraded. This has motivated lots of studies of the robust filtering technique for systems with any allowed uncertainties (see [2-6]).

It is well known that robust finite-horizon kalman filters could provide a good transient performance for the filtering system where modeling parameters and the statistics of noises are uncertainties. The objective of robust finite-horizon kalman filter is the design of a filter having an estimation error variance with an optimized guaranteed upper bound for dynamic systems with all allowed uncertainties. In the past few years, several results on this topic have been reported in the literature (see [7-10]). In [7], the finite-horizon Kalman filter for systems involving a norm-bounded uncertain block has been proposed and the performance of filter is improved by using multiple scaling parameters. In [8], a finite and infinite horizon robust kalman filter addressed the problem of estimating state variables for discrete-time systems with time-varying norm-bounded uncertainties in both the state and output matrices has been presented. In [9], a robust finite-horizon kalman filtering problem for linear discrete time-varying uncertain systems with additive and multiplicative noises is solved and an optimization approach based on the solutions to discrete Riccati difference equations is used. In [10], Dong et.al proposed a robust finite-horizon kalman filter for uncertainties discrete time-varying systems with uncertain-covariance white noises. However, all of these algorithms have assumed that there not exist state-delay and uncertainty in its matrix for dynamic systems. In reality, the delay is one of the main sources of instability in control systems.

In this note, we are concerned with the robust finite-horizon robust kalman filtering 
problem for uncertainty discrete time-varying systems with constant delay both in state and output matrices subject to norm-bounded uncertainties which is expected in the state, output and the white noise covariance matrices. The purpose is the design of a filter having an estimation error variance with an optimized guaranteed upper bound for all admissible uncertainties. We obtain the filter parameters state, state-delay and gain matrices are optimized to give a minimal upper bound on the state estimation error covariance for all admissible uncertainties. If the system modeling without uncertainties and state-delay, the proposed filter will reduce to the conventional Kalman filter.

The remainder of this paper is organized as follow. In Section II, the robust kalman filtering problem for uncertainties discrete time-varying systems with state-delay in state and output matrices is formulated. In Section III, the existence of upper bound on the estimation error covariance is studied. In Section IV, the robust finite-horizon kalman filter is designed and the filter parameters are optimized to give a minimal upper bound on the state estimation error covariance. In Section V, a numerical example is given to show the applicability of the robust finite-horizon kalman filter. At last, some conclusions will be concluded in Section VI.

\section{Problem Formulation}

Consider a following class of discrete time-varying uncertain system with state delay in state and output matrices defined on $k \in[0, N]$

$$
\begin{aligned}
& x_{k+1}=\left(A_{k}+H_{1, k} F_{k} E_{1, k}\right) x_{k}+\left(D_{k-\tau}+H_{3, k} F_{k} E_{3, k}\right) x_{k-\tau}+\left(B_{k}+H_{1, k} F_{k} E_{2, k}\right) w_{k} \\
& y_{k}=\left(C_{k}+H_{2, k} F_{k} E_{1, k}\right) x_{k}+\left(G_{k-\tau}+H_{4, k} F_{k} E_{3, k}\right) x_{k-\tau}+\left(L_{k}+H_{2, k} F_{k} E_{2, k}\right) v_{k} \\
& x_{k-\tau}=0, k \in[0, \tau]
\end{aligned}
$$

where $x_{k} \in R^{n}$ is the state, $x_{k-\tau} \in R^{n}$ is the state-delay, $y_{k} \in R^{p}$ is the measured output, $w_{k} \in R^{q 1}$ is the process noise, $v_{k} \in R^{q 2}$ is the measurement noise, $A_{k}, D_{k-\tau}$, $B_{k}, C_{k}, G_{k-\tau}, L_{k}, H_{1, k}, H_{2, k}, H_{3, k}, H_{4, k}, E_{1, k}, E_{2, k}$ and $E_{3, k}$ are known real time-varying matrices with appropriate dimensions, where $F_{k} \in R^{i \times j}$ is the norm-bounded time-varying uncertainty, i.e.

$F_{k}^{T} F_{k} \leq I, \forall k \in[0, N]$

The parameter uncertainty $F_{k}$ is said to be admissible if it satisfies (2).

The noise signals $w_{k}, v_{k}$ are Gaussian white noise sequences. Supposing $w_{k}, v_{k}$ and the initial state $x_{0}$ have the following statistical properties:

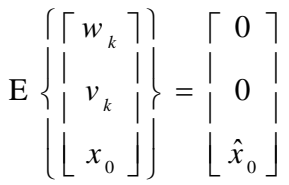

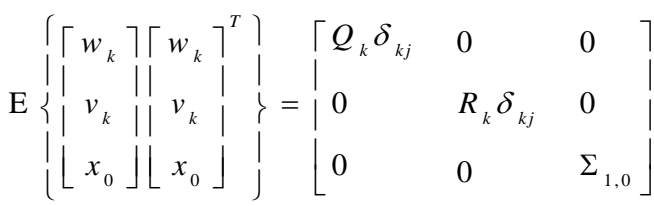

where $\mathrm{E}\{\bullet\}$ stands for the mathematical expectation operator, $Q_{k}, R_{k}$ and $\Sigma_{1,0}$ 
represent covariance matrices of noises and the initial state, and $\delta_{k j}$ denotes the Kronecker delta function, which is equal to unity for $k=j$ and zero elsewhere.

The required filter form for the uncertain system (1) is represented as

$$
\begin{aligned}
& \hat{x}_{k+1}=A_{o k} \hat{x}_{k}+D_{o k-\tau} \hat{x}_{k-\tau}+K_{o k}\left(y_{k}-C_{k} \hat{x}_{k}-G_{k} \hat{x}_{k-\tau}\right) \\
& \hat{x}_{k-\tau}=0, k \in[0, \tau]
\end{aligned}
$$

where $k \in[0, N], \quad \hat{x}_{k} \in R^{n}$ is the estimated state value and $\hat{x}_{k-\tau} \in R^{n}$ is the estimated state-delay value, $A_{o k}, D_{o k}$ and $K_{o k}$ are the filter parameters to be determined.

In next section, our goal is to prove the existence of an upper-bounded on the estimation error covariance and find a sequence of positive-definite matrices $\Xi_{k}$ satisfying

$$
\mathrm{E}\left[\left(x_{k}-\hat{x}_{k}\right)\left(x_{k}-\hat{x}\right)^{T}\right] \leq \Xi_{k}
$$

\section{Etimation Error Covarianceand Upper Bounds}

Since it is difficult to get the accurate error covariance for the system (1) because it involves uncertain and stochastic terms, therefore, we need to derive the estimation error covariance and obtain a corresponding upper bound. For this purpose, an augment state vector is defined

$$
\tilde{x}_{k}=\left[\begin{array}{l}
x_{k} \\
\hat{x}_{k}
\end{array}\right]
$$

and then the augmented state-space model system following form system (1) and filter (5) can be described as

$$
\tilde{x}_{k+1}=\left(\tilde{A}_{k}+\tilde{H}_{1, k} F_{k} \tilde{E}_{1, k}\right) \tilde{x}_{k}+\left(\tilde{D}_{k-\tau}+\tilde{H}_{3, k} F_{k} \tilde{E}_{3, k}\right) \tilde{x}_{k-\tau}+\left(\tilde{B}_{k}+\tilde{H}_{2, k} F_{k} \tilde{E}_{2, k}\right) \tilde{w}_{k}
$$

where

$$
\begin{aligned}
& \tilde{A}_{k}=\left[\begin{array}{cc}
A_{k} & 0 \\
K_{o k} C_{k} & A_{o k}-K_{o k} C_{k}
\end{array}\right], \tilde{H}_{1, k}=\left[\begin{array}{l}
H_{1, k} \\
K_{o k} H_{2, k}
\end{array}\right], \tilde{E}_{1, k}=\left[\begin{array}{ll}
E_{1, k} & 0
\end{array}\right] \\
& \tilde{D}_{k-\tau}=\left[\begin{array}{cc}
D_{k-\tau} & 0 \\
K_{o k} G_{k-\tau} & D_{o k-\tau}-K_{o k} G_{k-\tau}
\end{array}\right], \tilde{H}_{3, k}=\left\lceil\begin{array}{l}
H_{3, k} \\
K_{o k} H_{4, k}
\end{array}\right], \tilde{E}_{3, k}=\left[\begin{array}{ll}
E_{3, k} & 0
\end{array}\right] \\
& \tilde{B}_{k}=\left[\begin{array}{cc}
B_{k} & 0 \\
0 & K_{o k} L_{k}
\end{array}\right], \tilde{H}_{2, k}=\left[\begin{array}{ll}
H_{1, k} & 0 \\
0 & K_{o k} H_{2, k}
\end{array}\right] \tilde{E}_{2, k}=\left[\begin{array}{ll}
E_{2, k} & 0 \\
0 & E_{2, k}
\end{array}\right], \tilde{w}_{k}=\left\lceil\begin{array}{c}
w_{k} \\
v_{k}
\end{array}\right]
\end{aligned}
$$

Denote the state covariance matrices of the augmented system (8) as follow

$$
\left.\tilde{\Sigma}_{k}=\mathrm{E}\left[\tilde{x}_{k} \tilde{x}_{k}^{T}\right]=\mathrm{E}\left\{\begin{array}{l}
\left\lceil x_{k}\right\rceil\left\lceil x_{k}\right\rceil^{T} \\
\mid \hat{x}_{k}
\end{array}\right]\left[\begin{array}{l}
\hat{x}_{k}
\end{array}\right]\right\}
$$

According to (8) and (9), then the Lyapunov equation that governs its evolution can be defined as 


$$
\begin{aligned}
\tilde{\Sigma}_{k+1}= & \left(\tilde{A}_{k}+\tilde{H}_{1, k} F_{k} \tilde{E}_{1, k}\right) \tilde{\Sigma}_{k}\left(\tilde{A}_{k}+\tilde{H}_{1, k} F_{k} \tilde{E}_{1, k}\right)^{T} \\
& +\left(\tilde{A}_{k}+\tilde{H}_{1, k} F_{k} \tilde{E}_{1, k}\right) \tilde{\Sigma}_{k, k-\tau}\left(\tilde{D}_{k-\tau}+\tilde{H}_{3, k} F_{k} \tilde{E}_{3, k}\right)^{T} \\
& +\left(\tilde{D}_{k-\tau}+\tilde{H}_{3, k} F_{k} \tilde{E}_{3, k}\right) \tilde{\Sigma}_{k-\tau, k}\left(\tilde{A}_{k}+\tilde{H}_{1, k} F_{k} \tilde{E}_{1, k}\right)^{T} \\
& +\left(\tilde{D}_{k-\tau}+\tilde{H}_{3, k} F_{k} \tilde{E}_{3, k}\right) \tilde{\Sigma}_{k-\tau}\left(\tilde{D}_{k-\tau}+\tilde{H}_{3, k} F_{k} \tilde{E}_{3, k}\right)^{T} \\
& +\left(\tilde{B}_{k}+\tilde{H}_{2, k} F_{k} \tilde{E}_{2, k}\right) \tilde{W}_{k}\left(\tilde{B}_{k}+\tilde{H}_{2, k} F_{k} \tilde{E}_{2, k}\right)^{T} \\
\tilde{\Sigma}_{k-\tau}= & 0, \quad k \in[0, \tau]
\end{aligned}
$$

where

$$
\begin{aligned}
& \tilde{\Sigma}_{k, k-\tau}=\mathrm{E}\left[\tilde{x}_{k} \tilde{x}_{k-\tau}^{T}\right]=\mathrm{E}\left\{\left\{\begin{array}{l}
\left\lceil x_{k}\right\rceil\left\lceil x_{k-\tau}\right]^{T} \\
\hat{x}_{k}
\end{array}\right]\left[\begin{array}{l}
\hat{x}_{k-\tau}
\end{array}\right]\right\} \\
& \tilde{\Sigma}_{k, k-\tau}=0, k \in[0, \tau] \\
& \tilde{\Sigma}_{k-\tau, k}=\tilde{\Sigma}_{k, k-\tau}^{T}, \quad \tilde{W}_{k}=\left[\begin{array}{cc}
Q_{k} & 0 \\
0 & R_{k}
\end{array}\right]
\end{aligned}
$$

In order to test the existence the upper bound of the error covariance in (10), we will make use of the following lemma.

Lemma 1[11] Given any real matrices $\Sigma_{1}, \Sigma_{2}$ and $\Sigma_{3}$ with appropriate dimensions such that $\Sigma_{3}^{T} \Sigma_{3} \leq I$ and $\lambda>0$, then the following inequality holds:

$$
\Sigma_{1} \Sigma_{3} \Sigma_{2}+\Sigma_{2}^{T} \Sigma_{3}^{T} \Sigma_{1}^{T} \leq \lambda \Sigma_{1} \Sigma_{1}^{T}+\lambda^{-1} \Sigma_{2}^{T} \Sigma_{2} .
$$

Lemma2[12] Given matrices $A, H, E$ and $F$ with compatible dimensions such that $F F^{T} \leq I$. Let $X$ be a symmetric positive-definite matrix and there exist arbitrary $\alpha>0$ such that $\alpha^{-1} I-E X E^{T}>0$, then the following inequality holds:

$$
\begin{aligned}
(A+H F E) & X(A+H F E)^{T} \\
\leq & A\left(X^{-1}-\alpha E^{T} E\right)^{-1} A^{T}+\alpha^{-1} H H^{T} .
\end{aligned}
$$

Making use of the structure

$$
\begin{aligned}
& \left(\tilde{A}_{k}+\tilde{H}_{1, k} F \tilde{E}_{1, k}\right) \tilde{\Sigma}_{k, k-\tau}\left(\tilde{D}_{k-\tau}+\tilde{H}_{3, k} F \tilde{E}_{3, k}\right)^{T} \\
& +\left(\tilde{D}_{k-\tau}+\tilde{H}_{3, k} F \tilde{E}_{3, k}\right) \tilde{\Sigma}_{k-\tau, k}\left(\tilde{A}_{k}+\tilde{H}_{1, k} F \tilde{E}_{1, k}\right)^{T}
\end{aligned}
$$

which is similar to the structure in lemma 1 and making use of the structure

$$
\begin{aligned}
& \left(\tilde{A}_{k}+\tilde{H}_{1, k} F \tilde{E}_{1, k}\right) \tilde{\Sigma}_{k}\left(\tilde{A}_{k}+\tilde{H}_{1, k} F \tilde{E}_{1, k}\right)^{T} \\
& +\left(\tilde{D}_{k-\tau}+\tilde{H}_{3, k} F \tilde{E}_{3, k}\right) \tilde{\Sigma}_{k-\tau}\left(\tilde{D}_{k-\tau}+\tilde{H}_{3, k} F \tilde{E}_{3, k}\right)^{T}
\end{aligned}
$$

which is similar to the structure in lemma 2 and using the approach in [6] and [7], then the following result can be proved.

Theorem 1: Assume that there exists arbitrary $\alpha_{k}>0$ such that $\alpha_{k}^{-1} I-\tilde{E}_{1, k} \tilde{\Sigma}_{k} \tilde{E}_{1, k}^{T}>0$, arbitrary $\gamma_{k}>0 \quad$ such that $\gamma_{k}^{-1} I-\tilde{E}_{3, k} \tilde{\Sigma}_{k-\tau} \tilde{E}_{3, k}^{T}>0$ and arbitrary $\beta_{k}>0$ such that $\beta_{k}^{-1} I-\tilde{E}_{2, k} \tilde{W}_{k} \tilde{E}_{2, k}^{T}>0$, then 


$$
\begin{aligned}
& \tilde{\Sigma}_{k+1} \leq(1+\lambda) \tilde{A}_{k}\left(\tilde{\Sigma}_{k}^{-1}-\alpha_{k} \tilde{E}_{1, k}^{T} \tilde{E}_{1, k}\right)^{-1} \tilde{A}_{k}^{T} \\
& +\left(1+\lambda^{-1}\right) \tilde{D}_{k-\tau}\left(\tilde{\Sigma}_{k-\tau}^{-1}-\gamma_{k} \tilde{E}_{3, k}^{T} \tilde{E}_{3, k}\right)^{-1} \tilde{D}_{k-\tau}^{T} \\
& \tilde{B}_{k}\left(\tilde{W}_{k}^{-1}-\tilde{B}_{k} \tilde{E}_{2, k}^{T} \tilde{E}_{2, k}\right)^{-1} \tilde{B}_{k}^{T}+(1+\lambda) \alpha_{k}^{-1} \tilde{H}_{1, k} \tilde{H}_{1, k}^{T} \\
& +\left(1+\lambda^{-1}\right) \gamma_{k}^{-1} \tilde{H}_{3, k} \tilde{H}_{3, k}^{T}+\beta_{k}^{-1} \tilde{H}_{2, k} \tilde{H}_{2, k}^{T} \\
& \tilde{\Sigma}_{k-\tau}=0, k \in[0, \tau]
\end{aligned}
$$

with initial value $\tilde{\Sigma}_{0}=\left[\begin{array}{cc}P_{0} & 0 \\ 0 & 0\end{array}\right]$ and let

$\Sigma_{k+1}=(1+\lambda) \tilde{A}_{k}\left(\Sigma_{k}^{-1}-\alpha_{k} \tilde{E}_{1, k}^{T} \tilde{E}_{1, k}\right)^{-1} \tilde{A}_{k}^{T}$

$+\left(1+\lambda^{-1}\right) \tilde{D}_{k-\tau}\left(\Sigma_{k k-\tau}^{-1}-\gamma_{k} \tilde{E}_{3, k}^{T} \tilde{E}_{3, k}\right)^{-1} \tilde{D}_{k-1}^{T}$

$\tilde{B}_{k}\left(\tilde{W}_{k}^{-1}-\beta_{k} \tilde{E}_{2, k}^{T} \tilde{E}_{2, k}\right)^{-1} \tilde{B}_{k}^{T}+(1+\lambda) \alpha_{k}^{-1} \tilde{H}_{1, k} \tilde{H}_{1, k}^{T}$

$+\left(1+\lambda^{-1}\right) \gamma_{k}^{-1} \tilde{H}_{3, k} \tilde{H}_{3, k}^{T}+\beta_{k}^{-1} \tilde{H}_{2, k} \tilde{H}_{2, k}^{T}$

$\Sigma_{k-\tau}=0, k \in[0, \tau]$

$$
\begin{aligned}
& \alpha_{k}^{-1} I-\tilde{E}_{1, k} \Sigma_{k} \tilde{E}_{1, k}^{T}>0 \\
& \gamma_{k}^{-1} I-\tilde{E}_{3, k} \Sigma_{k-\tau} \tilde{E}_{3, k}^{T}>0 \\
& \beta_{k}^{-1} I-\tilde{E}_{2, k} \tilde{W}_{k} \tilde{E}_{2, k}^{T}>0
\end{aligned}
$$

with initial value $\Sigma_{0}=\tilde{\Sigma}_{0}$, then $\tilde{\Sigma}_{k} \leq \Sigma_{k}$ and $\Sigma_{k}$ will be a candidate upper-bound for the estimation error covariance $\tilde{\Sigma}_{k}$.

It is obviously that the solution of (14) is not unique in general. Hence, in the next section, we will try to find a proper solution and solve the filter parameters $A_{o k}, D_{o k-\tau}$ and $K_{o k}$ to minimize $\operatorname{tr}\left(\Xi_{k}\right)$.

\section{Robust Finite-Horizon Filter Design}

In this section, our objective is twofold. One is to find a solution of (12), and other is to design a filter that minimizes the upper bound of the actual state estimation error variance. Hence, we propose the following theorem.

Theorem 2: given the sequence of positive scalars $\alpha_{k}, \gamma_{k}$ and $\beta_{k}$ satisfying (13), (14) and (15), then the solution to (12) will be of the following form

$$
\Sigma_{k}=\left[\begin{array}{cc}
\Sigma_{1, k} & \Sigma_{1, k}-\Xi_{k} \\
\Sigma_{1, k}-\Xi_{k} & \Sigma_{1, k}-\Xi_{k}
\end{array}\right]
$$

where $\Sigma_{1, k}$ and $\Xi_{k}$ are defined as (22) and (23)

and $\operatorname{tr}\left(\Xi_{k}\right)$ is minimizes if the parameters of filter(5) are of the form:

$$
\begin{aligned}
A_{o k}= & A_{k}+\left(A_{k}-K_{o k} C_{k}\right) \Xi_{k} E_{1, k}^{T}\left(\alpha^{-1} I-E_{1, k} \Xi_{k} E_{1, k}^{T}\right)^{-1} E_{1, k} \\
D_{o k-\tau}= & D_{k-\tau}+\left(D_{k-\tau}-K_{o k} G_{k-\tau}\right) \Xi_{k-\tau} E_{3, k}^{T}\left(\gamma^{-1} I-E_{3, k} \Xi_{k-\tau} E_{3, k}^{T}\right)^{-1} E_{3, k} \\
K_{o k}= & {\left[(1+\lambda) A_{k}\left(\Xi_{k}^{-1}-\alpha_{k} E_{1, k}^{T} E_{1, k}\right)^{-1} C_{k}^{T}+\left(1+\lambda^{-1}\right) D_{k-\tau}\left(\Xi_{k-\tau}^{-1}-\gamma_{k-\tau} E_{3, k}^{T} E_{3,}\right) \mathrm{G}_{k-\tau}^{T}\right.} \\
& \left.\quad+(1+\lambda) H_{1, k} H_{2, k}^{T}+\left(1+\lambda^{-1}\right) H_{3, k} H_{4, k}^{T}\right] R_{1, k}^{-1}
\end{aligned}
$$

where 


$$
\begin{aligned}
R_{1, k}= & \left((1+\lambda) C_{k}\left(\Xi_{k}^{-1}-\alpha_{k} E_{1, k}^{T} E_{1, k}\right)^{-1} C_{k}^{T}\right. \\
& +\left(1+\lambda^{-1}\right) G_{k-\tau}\left(\Xi_{k}^{-1}-\gamma_{k} E_{3, k}^{T} E_{3, k}\right)^{-1} G_{k-\tau}^{T} \\
& +L_{k}\left(R_{k}^{-1}-\beta_{k} E_{2, k}^{T} E_{2, k}\right)^{-1} L_{k}^{T}+\left((1+\lambda) \alpha_{k}^{-1}\right. \\
& \left.\left.+\beta_{k}^{-1}\right) H_{2, k} H_{2, k}^{T}+\left(1+\lambda^{-1}\right) \gamma_{k}^{-1} H_{4, k} H_{4, k}^{T}\right)
\end{aligned}
$$

and the state covariance will be

$$
\begin{aligned}
\Sigma_{1, k+1}= & (1+\lambda) A_{k}\left(\Sigma_{1, k}^{-1}-\alpha_{k} E_{1, k}^{T} E_{1, k}\right)^{-1} A_{k}^{T} \\
& +\left(1+\lambda^{-1}\right) D_{k-\tau}\left(\Sigma_{1, k-\tau}^{-1}-\gamma_{k} E_{3, k}^{T} E_{3, k}\right)^{-1} D_{k-\tau}^{T} \\
& +B_{k}\left(Q_{k}^{-1}-\beta_{k} E_{2, k}^{T} E_{2, k}\right)^{-1} B_{k}^{T}+\left(1+\lambda^{-1}\right) \gamma_{k}^{-1} \\
& \bullet H_{3, k} H_{3, k}^{T}+\left((1+\lambda) \alpha_{k}^{-1}+\beta_{k}^{-1}\right) H_{1, k} H_{1, k}^{T}
\end{aligned}
$$

and the estimation error covariance will be

$$
\begin{aligned}
& \Xi_{k+1}=-\left[(1+\lambda) \alpha_{k}^{-1} H_{1, k} H_{2, k}^{T}+\left(1+\lambda^{-1}\right) \gamma_{k}^{-1} H_{3, k} H_{4, k}^{T}\right. \\
& +(1+\lambda) A_{k}\left(\Xi_{k}^{-1}-a_{k} E_{1, k}^{T} E_{1, k}\right)^{-1} C_{k}^{T}+\left(1+\lambda^{-1}\right) D_{k-\tau} \\
& \bullet \\
& \left.\bullet\left(\Xi_{k-\tau}^{-1}-\gamma_{k} E_{3, k}^{T} E_{3, k}\right)^{-1} G_{k-\tau}^{T}\right] R_{1, k}^{-1}\left[(1+\lambda) \alpha_{k}^{-1} H_{2, k} H_{1, k}^{T}\right. \\
& +\left(1+\lambda^{-1}\right) \gamma_{k}^{-1} H_{4, k} H_{3, k}^{T}+(1+\lambda) C_{k}\left(\Xi_{k}^{-1}-a_{k} E_{1, k}^{T} E_{1, k}\right)^{-1} A_{k}^{T} \\
& \left.+\left(1+\lambda^{-1}\right) G_{k-\tau}\left(\Xi_{k-\tau}^{-1}-\gamma_{k} E_{3, k}^{T} E_{3, k}\right)^{-1} D_{k-\tau}^{T}\right]+(1+\lambda) A_{k} \\
& \bullet\left(\Xi_{k}^{-1}-a_{k} E_{1, k}^{T} E_{1, k}\right)^{-1} A_{k}^{T}+\left(1+\lambda^{-1}\right) D_{k-\tau}\left(\Xi_{k-\tau}^{-1}\right. \\
& \left.-\gamma_{k} E_{3, k}^{T} E_{3, k}\right)^{-1} D_{k-\tau}^{T}+B_{k}\left(Q_{k}^{-1}-\beta_{k} E_{2, k}^{T} E_{2, k}\right)^{-1} B_{k}^{T} \\
& +\left((1+\lambda) \alpha_{k}^{-1}+\beta_{k}^{-1}\right) H_{1, k} H_{1, k}^{T}+\left(1+\lambda^{-1}\right) \gamma_{k}^{-1} H_{3, k} H_{3, k}^{T}
\end{aligned}
$$

Proof: To show that (16) is a solution to (12), substituting filter parameter expressions (16), (17), (18) and (19) into the right-hand side of (12) and considering conditions (20) and (21), straightforward algebraic manipulations show that the right-hand side of (12) is given by

$$
\begin{aligned}
& (1+\lambda) \tilde{A}_{k}\left(\Sigma_{k}^{-1}-\alpha_{k} \tilde{E}_{1, k}^{T} \tilde{E}_{1, k}\right)^{-1} \tilde{A}_{k}^{T}+ \\
& +\left(1+\lambda^{-1}\right) \tilde{D}_{k-\tau}\left(\Sigma_{k k-\tau}^{-1}-\gamma_{k} \tilde{E}_{3, k-\tau}^{T} \tilde{E}_{3, k-\tau}\right)^{-1} \tilde{D}_{k-1}^{T} \\
& \tilde{B}_{k}\left(\tilde{W}_{k}^{-1}-\beta_{k} \tilde{E}_{2, k}^{T} \tilde{E}_{2, k}\right)^{-1} \tilde{B}_{k}^{T}+(1+\lambda) \alpha_{k}^{-1} \tilde{H}_{1, k} \tilde{H}_{1, k}^{T} \\
& +\left(1+\lambda^{-1}\right) \gamma_{k}^{-1} \tilde{H}_{3, k-\tau} \tilde{H}_{3, k-\tau}^{T}+\beta_{k}^{-1} \tilde{H}_{2, k} \tilde{H}_{2, k}^{T} \\
& =\left[\begin{array}{cc}
\Sigma_{1, k+1} & \Sigma_{1, k+1}-\Xi_{k+1} \\
\Sigma_{1, k+1}-\Xi_{k+1} & \Sigma_{1, k+1}-\Xi_{k+1}
\end{array}\right]
\end{aligned}
$$

This means that (16) is a solution to (12).

In the following part, we need to prove that upper bound is optimized by using parameters $A_{o k}, D_{o k-\tau}$ and $K_{o k}$. According to (12) and (16), we have 


$$
\begin{aligned}
\Xi_{k+1}= & {\left[\begin{array}{ll}
I & -I
\end{array}\right] \Sigma_{K+1}\left[\begin{array}{ll}
I & -I
\end{array}\right]^{T} } \\
= & (1+\lambda)\left[\begin{array}{ll}
A_{k}-K_{o k} C_{k} & K_{o k} C_{k}-A_{o k}
\end{array}\right] \\
& \bullet\left(\Sigma_{k}^{-1}-\alpha_{k} \tilde{E}_{1, k}^{T} \tilde{E}_{1, k}\right)^{-1} \square\left[\begin{array}{lll}
A_{k}-K_{o k} C_{k} & K_{o k} C_{k}-A_{o k}
\end{array}\right]^{T} \\
& +\left(1+\lambda^{-1}\right)\left[\begin{array}{lll}
D_{k-\tau}-K_{o k} G_{k-\tau} & K_{o k} G_{k-\tau}-D_{o k-\tau}
\end{array}\right] \\
& \bullet\left(\Sigma_{k-\tau}^{-1}-\gamma_{k} \tilde{E}_{3, k}^{T} \tilde{E}_{3, k}\right)^{-1}\left[\begin{array}{ll}
D_{k-\tau}-K_{o k} G_{k-\tau} & K_{o k} G_{k-\tau}-D_{o k-\tau}
\end{array}\right]^{T} \\
& +\left[\begin{array}{lll}
B_{k} & -K_{o k} L_{k}
\end{array}\right]\left(\tilde{W}_{k}^{-1}-\beta_{k} \tilde{E}_{2, k}^{T} \tilde{E}_{2, k}\right)^{-1}\left[\begin{array}{ll}
B_{k} & -K_{o k} L_{k}
\end{array}\right]^{T} \\
& +(1+\lambda) \alpha_{k}^{-1}\left(H_{1, k}-K_{o k} H_{2, k}\right)\left(H_{1, k}-K_{o k} H_{2, k}\right)^{T} \\
& +\left(1+\lambda^{-1}\right) \gamma_{k}^{-1}\left(H_{3, k}-K_{o k} H_{4, k}\right)\left(H_{3, k}-K_{o k} H_{4, k}\right)^{T} \\
& +\beta_{k}^{-1}\left[H_{1, k}-K_{o k} H_{2, k}\right]\left[\begin{array}{ll}
H_{1, k} & -K_{o k} H_{2, k}
\end{array}\right]^{T}
\end{aligned}
$$

Obviously, $\Xi_{k+1}$ is related to $A_{o k}, D_{o k-\tau}$ and $K_{o k}$. In order to minimized the estimation error covariance upper bound $\Xi_{k+1}$, we must find optimal filter parameters $A_{o k}$, $D_{o k-\tau}$ and $K_{o k}$. So we take the first variation to (23) with respect to $A_{o k}, D_{o k-\tau}$ and $K_{o k}$ and equal them to zero

$$
\begin{aligned}
& \frac{\partial \Xi_{k+1}}{\partial A_{o k}}=\left[A_{k}-K_{o k} C_{k} \quad K_{o k} C_{k}-A_{o k}\right]\left(\Sigma_{k}^{-1}-\alpha_{k} \tilde{E}_{1, k}^{T} \tilde{E}_{1, k}\right)^{-1}\left[\begin{array}{ll}
0 & -I
\end{array}\right]^{T}=0 \\
& \frac{\partial \Xi_{k+1}}{\partial D_{o k-\tau}}=\left[D_{k-\tau}-K_{o k} G_{k-\tau} \quad K_{o k} G_{k-\tau}-D_{o k-\tau}\right]\left(\Sigma_{k-\tau}^{-1}-\gamma_{k} \tilde{E}_{3, k}^{T} \tilde{E}_{3, k}\right)^{-1}\left[\begin{array}{ll}
0 & -I
\end{array}\right]^{T}=0 \\
& \frac{\partial \Xi_{k+1}}{\partial K_{o k}}=(1+\lambda)\left[A_{k}-K_{o k} C_{k} \quad K_{o k} C_{k}-A_{o k}\right] \\
& \bullet\left(\Sigma_{k}^{-1}-\alpha_{k} \tilde{E}_{1, k}^{T} \tilde{E}_{1, k}\right)^{-1}\left[\begin{array}{ll}
-C_{k} & C_{k}
\end{array}\right]^{T} \\
& +\left(1+\lambda^{-1}\right)\left[D_{k-\tau}-K_{o k} G_{k-\tau} \quad K_{o k} G_{k-\tau}-D_{o k-\tau}\right] \\
& \bullet\left(\Sigma_{k}^{-1}-\gamma_{k} \tilde{E}_{3, k}^{T} \tilde{E}_{3, k}\right)^{-1}\left[\begin{array}{ll}
-G_{k-\tau} & G_{k-\tau}
\end{array}\right]^{T}+\left[\begin{array}{ll}
B_{k} & -K_{o k} L_{k}
\end{array}\right] \\
& \text { - }\left(\tilde{W}_{k}^{-1}-\beta_{k} \tilde{E}_{2, k}^{T} \tilde{E}_{2, k}\right)^{-1}\left[\begin{array}{ll}
0 & -L_{k}
\end{array}\right]^{T}+(1+\lambda) \alpha_{k}^{-1}\left(H_{1, k}\right. \\
& \left.-K_{o k} H_{2, k}\right)\left(-H_{2, k}\right)^{T}+\left(1+\lambda^{-1}\right) \gamma_{k}^{-1}\left(H_{3, k}-K_{o k} H_{4, k}\right)\left(-H_{4, k}\right)^{T} \\
& +\beta_{k}^{-1}\left[\begin{array}{ll}
H_{1, k} & -K_{o k} H_{2, k}
\end{array}\right]\left[\begin{array}{ll}
0 & -H_{2, k}
\end{array}\right]^{T}=0
\end{aligned}
$$

Denote that

$$
\left(\Sigma_{k}^{-1}-\alpha_{k} \tilde{E}_{1, k}^{T} \tilde{E}_{1, k}\right)^{-1}=\left[\begin{array}{ll}
S_{1} & S_{2} \\
S_{3} & S_{4}
\end{array}\right]
$$

Using the lemma of matrix inversion [13] and (16), we have

$$
\begin{gathered}
S_{1}=\Sigma_{1, k}+\Sigma_{1, k} E_{1, k}^{T}\left(\alpha_{k}^{-1} I-E_{1, k} \Sigma_{1, k} E_{1, k}^{T}\right)^{-1} E_{1, k} \Sigma_{1, k} \\
S_{2}=\left(\Sigma_{1, k}-\Xi_{k}\right)+\Sigma_{1, k} E_{1, k}^{T}\left(\alpha_{k}^{-1} I-E_{1, k} \Sigma_{1, k} E_{1, k}^{T}\right)^{-1} E_{1, k}\left(\Sigma_{1, k}-\Xi_{k}\right) \\
S_{3}=\left(\Sigma_{1, k}-\Xi_{k}\right)+\left(\Sigma_{1, k}-\Xi_{k}\right) E_{1, k}^{T}\left(\alpha_{k}^{-1} I-E_{1, k} \Sigma_{1, k} E_{1, k}^{T}\right)^{-1} E_{1, k} \Sigma_{1, k} \\
S_{4}=\left(\Sigma_{1, k}-\Xi_{k}\right)+\left(\Sigma_{1, k}-\Xi_{k}\right) E_{1, k}^{T}\left(\alpha_{k}^{-1} I-E_{1, k} \Sigma_{1, k} E_{1, k}^{T}\right)^{-1} E_{1, k}\left(\Sigma_{1, k}-\Xi_{k}\right)
\end{gathered}
$$

From (28), (29), (30) and (31), the following facts are obtained.

$$
\begin{aligned}
& {\left[\begin{array}{ll}
I & -I
\end{array}\right]\left(\Sigma_{k}^{-1}-\alpha_{k} \tilde{E}_{1, k}^{T} \tilde{E}_{1, k}\right)^{-1}\left[\begin{array}{ll}
0 & -I
\end{array}\right]^{T}=S_{3}-S_{1}} \\
& =\Xi_{k} E_{1, k}^{T}\left(\alpha_{k}^{-1} I-E_{1, k} \Sigma_{1, k} E_{1, k}^{T}\right)^{-1} E_{1, k}\left(\Xi_{k}-\Sigma_{1, k}\right)
\end{aligned}
$$


From (24), we have

$$
\begin{aligned}
& {\left[\begin{array}{ll}
0 & I
\end{array}\right]\left(\Sigma_{k}^{-1}-\alpha_{k} \tilde{E}_{1, k}^{T} \tilde{E}_{1, k}\right)^{-1}\left[\begin{array}{ll}
0 & -I
\end{array}\right]^{T}} \\
& =-S_{4} \\
& =\left(\Xi_{k}-\Sigma_{1, k}\right)-\left(\Xi_{k}-\Sigma_{1, k}\right) E_{1, k}^{T} \\
& \quad \quad \quad\left(\alpha_{k}^{-1} I-E_{1, k} \Sigma_{1, k} E_{1, k}^{T}\right)^{-1} E_{1, k}\left(\Xi_{k}-\Sigma_{1, k}\right)
\end{aligned}
$$

$$
\begin{aligned}
& {\left[A_{k}-K_{o k} C_{k} \quad K_{o k} C_{k}-A_{o k}\right]\left(\Sigma_{k}^{-1}-\alpha_{k} \tilde{E}_{1, k}^{T} \tilde{E}_{1, k}\right)^{-1}\left[\begin{array}{ll}
0 & -I
\end{array}\right]^{T}} \\
& =\left[\begin{array}{ll}
A_{k}-K_{o k} C_{k} & K_{o k} C_{k}-A_{k}
\end{array}\right]\left(\Sigma_{k}^{-1}-\alpha_{k} \tilde{E}_{1, k}^{T} \tilde{E}_{1, k}\right)^{-1}\left[\begin{array}{ll}
0 & -I
\end{array}\right]^{T} \\
& +\left[\begin{array}{ll}
0 & A_{k}-A_{o k}
\end{array}\right]\left(\Sigma_{k}^{-1}-\alpha_{k} \tilde{E}_{1, k}^{T} \tilde{E}_{1, k}\right)^{-1}\left[\begin{array}{ll}
0 & -I
\end{array}\right]^{T} \\
& =\left(A_{k}-K_{o k} C_{k}\right)\left[\begin{array}{ll}
I & -I
\end{array}\right]\left(\Sigma_{k}^{-1}-\alpha_{k} \tilde{E}_{1, k}^{T} \tilde{E}_{1, k}\right)^{-1}\left[\begin{array}{ll}
0 & -I
\end{array}\right]^{T} \\
& +\left(A_{k}-A_{o k}\right)\left[\begin{array}{ll}
0 & I
\end{array}\right]\left(\Sigma_{k}^{-1}-\alpha_{k} \tilde{E}_{1, k}^{T} \tilde{E}_{1, k}\right)^{-1}\left[\begin{array}{ll}
0 & -I
\end{array}\right]^{T}
\end{aligned}
$$

Substituting (32) and (33) into (34), then the optimal filter parameter $A_{o k}$ is given by

$$
\begin{aligned}
A_{o k}= & A_{k}+\left(A_{k}-K_{o k} C_{k}\right) \Xi_{k} E_{1, k}^{T}\left(\alpha_{k}^{-1} I-E_{1, k} \Sigma_{1, k} E_{1, k}^{T}\right)^{-1} \\
& \cdot E_{1, k}\left[I-\left(\Xi_{k}-\Sigma_{1, k}\right) E_{1, k}^{T}\left(\alpha_{k}^{-1} I-E_{1, k} \Sigma_{1, k} E_{1, k}^{T}\right)^{-1} E_{1, k}\right]^{-1} \\
= & A_{k}+\left(A_{k}-K_{o k} C_{k}\right) \Xi_{k} E_{1, k}^{T}\left(\alpha_{k}^{-1} I-E_{1, k} \Xi_{k} E_{1, k}^{T}\right)^{-1} E_{1, k}
\end{aligned}
$$

Thanks to the structure $D_{o k-\tau}$ which is similar to the structure $A_{o k}$, so we can solve $D_{o k-\tau}$ according to the way of solving $A_{o k}$.

Denote that

$$
\left(\Sigma_{k-\tau}^{-1}-\gamma_{k} \tilde{E}_{3, k}^{T} \tilde{E}_{3, k}\right)^{-1}=\left[\begin{array}{ll}
T_{1} & T_{2} \\
T_{3} & T_{4}
\end{array}\right]
$$

Using the lemma of matrix inversion and (12), then we have

$$
\begin{aligned}
T_{1}= & \Sigma_{1, k-\tau}+\Sigma_{1, k-\tau} E_{3, k}^{T}\left(\gamma_{k}^{-1} I\right. \\
& \left.-E_{3, k} \Sigma_{1, k-\tau} E_{3, k}^{T}\right)^{-1} E_{3, k} \Sigma_{1, k-\tau} \\
T_{2}= & \left(\Sigma_{1, k-\tau}-\Xi_{k-\tau}\right)+\Sigma_{1, k-\tau} E_{3, k}^{T}\left(\gamma_{k}^{-1} I\right. \\
& \left.-E_{3, k} \Sigma_{1, k-\tau} E_{3, k}^{T}\right)^{-1} E_{3, k}\left(\Sigma_{1, k-\tau}-\Xi_{k-\tau}\right) \\
T_{3}= & \left(\Sigma_{1, k-\tau}-\Xi_{k-\tau}\right)+\left(\Sigma_{1, k-\tau}-\Xi_{k-\tau}\right) E_{3, k}^{T} \\
& \bullet\left(\gamma_{k}^{-1} I-E_{3, k} \Sigma_{1, k-\tau} E_{3, k}^{T}\right)^{-1} E_{3, k} \Sigma_{1, k-\tau} \\
T_{4}= & \left(\Sigma_{1, k-\tau}-\Xi_{k-\tau}\right)+\left(\Sigma_{1, k-\tau}-\Xi_{k-\tau}\right) E_{3, k}^{T}\left(\gamma_{k}^{-1} I\right. \\
& \left.-E_{3, k} \Sigma_{1, k-\tau} E_{3, k}^{T}\right)^{-1} E_{3, k}\left(\Sigma_{1, k-\tau}-\Xi_{k-\tau}\right)
\end{aligned}
$$

From (37), (38), (39) and (40), we have the following facts

$$
\begin{aligned}
& {\left[\begin{array}{ll}
I & -I
\end{array}\right]\left(\Sigma_{k-\tau}^{-1}-\gamma_{k} \tilde{E}_{3, k}^{T} \tilde{E}_{3, k}\right)^{-1}\left[\begin{array}{ll}
0 & -I
\end{array}\right]^{T}=T_{3}-T_{1}} \\
& =\Xi_{k-\tau} E_{3, k}^{T}\left(\gamma_{k}^{-1} I-E_{3, k} \Sigma_{1, k-\tau} E_{3, k}^{T}\right)^{-1} E_{3, k}\left(\Xi_{k-\tau}-\Sigma_{1, k-\tau}\right) \\
& {\left[\begin{array}{ll}
0 & I
\end{array}\right]\left(\Sigma_{k-\tau}^{-1}-\gamma_{k} \tilde{E}_{3, k}^{T} \tilde{E}_{3, k}\right)^{-1}\left[\begin{array}{ll}
0 & -I
\end{array}\right]^{T}=-T_{4}} \\
& =\left(\Xi_{k-\tau}-\Sigma_{1, k-\tau}\right)-\left(\Xi_{k-\tau}-\Sigma_{1, k-\tau}\right) E_{3, k}^{T} \\
& \text { - }\left(\gamma_{k}^{-1} I-E_{3, k} \Sigma_{1, k-\tau} E_{3, k}^{T}\right)^{-1} E_{3, k}\left(\Xi_{k-\tau}-\Sigma_{1, k-\tau}\right)
\end{aligned}
$$

From (25), we have 


$$
\begin{aligned}
& {\left[\begin{array}{ll}
D_{k-\tau}-K_{o k} G_{k-\tau} & K_{o k} G_{k-\tau}-D_{o k-\tau}
\end{array}\right]} \\
& \bullet\left(\Sigma_{k-\tau}^{-1}-\gamma_{k} \tilde{E}_{3, k}^{T} \tilde{E}_{3, k}\right)^{-1}\left[\begin{array}{ll}
0 & -I
\end{array}\right]^{T} \\
& =\left[\begin{array}{ll}
D_{k-\tau}-K_{o k} G_{k-\tau} & K_{o k} G_{k-\tau}-D_{k-\tau}
\end{array}\right] \\
& \text { • }\left(\Sigma_{k-\tau}^{-1}-\gamma_{k} \tilde{E}_{3, k}^{T} \tilde{E}_{3, k}\right)^{-1}\left[\begin{array}{ll}
0 & -I
\end{array}\right]^{T} \\
& +\left[\begin{array}{ll}
0 & D_{k-\tau}-D_{o k-\tau}
\end{array}\right]\left(\Sigma_{k-\tau}^{-1}-\gamma_{k} \tilde{E}_{3, k}^{T} \tilde{E}_{3, k}\right)^{-1}\left[\begin{array}{ll}
0 & -I
\end{array}\right]^{T} \\
& =\left(D_{k-\tau}-K_{o k} G_{k-\tau}\right)\left[\begin{array}{ll}
I & -I
\end{array}\right]\left(\Sigma_{k-\tau}^{-1}-\gamma_{k} \tilde{E}_{3, k}^{T} \tilde{E}_{3, k}\right)^{-1}\left[\begin{array}{ll}
0 & -I
\end{array}\right]^{T} \\
& +\left(D_{k-\tau}-D_{o k-\tau}\right)\left[\begin{array}{ll}
0 & I
\end{array}\right]\left(\Sigma_{k-\tau}^{-1}-\gamma_{k} \tilde{E}_{3, k}^{T} \tilde{E}_{3, k}\right)^{-1}\left[\begin{array}{ll}
0 & -I
\end{array}\right]^{T}
\end{aligned}
$$

Substituting (41) and (42) into (43), then the optimal filter parameter $D_{o k-\tau}$ is given by

$$
\begin{aligned}
D_{o k-\tau}= & D_{k-\tau}+\left(D_{k-\tau}-K_{o k} G_{k-\tau}\right) \Xi_{k-\tau} E_{3, k}^{T}\left(\gamma_{k}^{-1} I\right. \\
- & \left.E_{3, k} \Sigma_{1, k-\tau} E_{3, k}^{T}\right)^{-1} E_{3, k}\left[I-\left(\Xi_{k-\tau}-\Sigma_{1, k-\tau}\right)\right. \\
& \left.\bullet E_{3, k}^{T}\left(\gamma_{k}^{-1} I-E_{3, k} \Sigma_{1, k-\tau} E_{3, k}^{T}\right)^{-1} E_{3, k}\right]^{-1} \\
= & D_{k-\tau}+\left(D_{k-\tau}-K_{o k} G_{k-\tau}\right) \Xi_{k-\tau} E_{3, k}^{T} \\
& \left(\gamma_{k}^{-1} I-E_{3, k} \Sigma_{1, k-\tau} E_{3, k}^{T}\right)^{-1} E_{3, k}
\end{aligned}
$$

When the optimal filter parameters $A_{o k}$ and $D_{o k-\tau}$ are determined, the last task is to solve the optimal filter parameter $K_{o k}$ from (26). According to (28), (29), (30), (31), (37), (38), (39) and (40), the following facts are obtained.

$$
\begin{aligned}
& {\left[\begin{array}{ll}
I & -I
\end{array}\right]\left(\Sigma_{k}^{-1}-\alpha_{k} \tilde{E}_{1, k}^{T} \tilde{E}_{1, k}\right)^{-1}\left[\begin{array}{ll}
-I & I
\end{array}\right]^{T}} \\
& =S_{2}+S_{3}-S_{1}-S_{4} \\
& =-\Xi_{k}-\Xi_{k} E_{1, k}^{T}\left(\alpha_{k}^{-1} I-E_{1, k} \Sigma_{1, k} E_{1, k}^{T}\right)^{-1} E_{1, k} \Xi_{k} \\
& {\left[\begin{array}{ll}
0 & I
\end{array}\right]\left(\Sigma_{k}^{-1}-\alpha_{k} \tilde{E}_{1, k}^{T} \tilde{E}_{1, k}\right)^{-1}\left[\begin{array}{ll}
-I & I
\end{array}\right]^{T}} \\
& =S_{4}-S_{3} \\
& =-\left(\Sigma_{1, k}-\Xi_{k}\right) E_{1, k}^{T}\left(\alpha_{k}^{-1} I-E_{1, k} \Sigma_{1, k} E_{1, k}^{T}\right)^{-1} E_{1, k} \Xi_{k} \\
& {\left[\begin{array}{ll}
I & -I
\end{array}\right]\left(\Sigma_{k-\tau}^{-1}-\gamma_{k} \tilde{E}_{3, k}^{T} \tilde{E}_{3, k}\right)^{-1}\left[\begin{array}{ll}
-I & I
\end{array}\right]^{T}} \\
& =T_{2}+T_{3}-T_{1}-T_{4} \\
& =-\Xi_{k-\tau}-\Xi_{k-\tau} E_{3, k}^{T}\left(\gamma_{k}^{-1} I-E_{3, k} \Sigma_{1, k-\tau} E_{3, k}^{T}\right)^{-1} E_{3, k} \Xi_{k-\tau} \\
& {\left[\begin{array}{ll}
0 & I
\end{array}\right]\left(\Sigma_{k-\tau}^{-1}-\gamma_{k} \tilde{E}_{3, k}^{T} \tilde{E}_{3, k}\right)^{-1}\left[\begin{array}{ll}
-I & I
\end{array}\right]^{T}} \\
& =T_{4}-T_{3} \\
& =-\left(\Sigma_{1, k-\tau}-\Xi_{k-\tau}\right) E_{3, k}^{T}\left(\gamma_{k}^{-1} I-E_{3, k} \Sigma_{1, k-\tau} E_{3, k}^{T}\right)^{-1} E_{3, k} \Xi_{k-\tau}
\end{aligned}
$$

From (26), we have 


$$
\begin{aligned}
& {\left[A_{k}-K_{o k} C_{k} \quad K_{o k} C_{k}-A_{o k}\right]\left(\Sigma_{k}^{-1}-\alpha_{k} \tilde{E}_{1, k}^{T} \tilde{E}_{1, k}\right)^{-1}}
\end{aligned}
$$

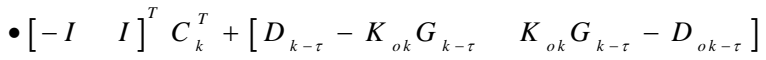

$$
\begin{aligned}
& \bullet\left(\Sigma_{k-\tau}^{-1}-\gamma_{k} \tilde{E}_{3, k}^{T} \tilde{E}_{3, k}\right)^{-1}\left[\begin{array}{ll}
-I & I
\end{array}\right]^{T} G_{k-\tau}^{T}
\end{aligned}
$$

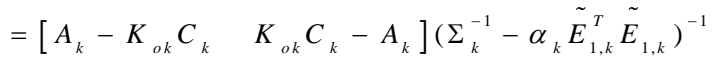

$$
\begin{aligned}
& \text { - }\left[\begin{array}{ll}
-I & I
\end{array}\right]^{T} C_{k}^{T}+\left[\begin{array}{ll}
0 & A_{k}-A_{o k}
\end{array}\right]\left(\Sigma_{k}^{-1}-\alpha_{k} \tilde{E}_{1, k}^{T} \tilde{E}_{1, k}\right)^{-1} \\
& \text { - }\left[\begin{array}{ll}
-I & I
\end{array}\right]^{T} C_{k}^{T}+\left[D_{k-\tau}-K_{o k} G_{k-\tau} \quad K_{o k} G_{k-\tau}-D_{o k-\tau}\right] \\
& \text { - }\left(\Sigma_{k-\tau}^{-1}-\gamma_{k} \tilde{E}_{3, k}^{T} \tilde{E}_{3, k}\right)^{-1}\left[\begin{array}{ll}
-I & I
\end{array}\right]^{T} G_{k-\tau}^{T} \\
& +\left[\begin{array}{ll}
0 & D_{k-\tau}-D_{o k-\tau}
\end{array}\right]\left(\Sigma_{k-\tau}^{-1}-\gamma_{k} \tilde{E}_{3, k}^{T} \tilde{E}_{3, k}\right)^{-1}\left[\begin{array}{ll}
-I & I
\end{array}\right]^{T} G_{k-\tau}^{T} \\
& =\left(A_{k}-K_{o k} C_{k}\right)\left[\begin{array}{ll}
I & -I
\end{array}\right]\left(\Sigma_{k}^{-1}-\alpha_{k} \tilde{E}_{1, k}^{T} \tilde{E}_{1, k}\right)^{-1} \\
& \text { - }\left[\begin{array}{ll}
-I & I
\end{array}\right]^{T} C_{k}^{T}+\left(A_{k}-A_{o k}\right)\left[\begin{array}{ll}
0 & I
\end{array}\right]\left(\Sigma_{k}^{-1}-\alpha_{k} \tilde{E}_{1, k}^{T} \tilde{E}_{1, k}\right)^{-1} \\
& \text { - }\left[\begin{array}{ll}
-I & I
\end{array}\right]^{T} C_{k}^{T}+\left(D_{k-\tau}-K_{o k} G_{k-\tau}\right)\left[\begin{array}{ll}
I & -I
\end{array}\right]\left(\Sigma_{k}^{-1}\right. \\
& \left.-\alpha_{k} \tilde{E}_{1, k}^{T} \tilde{E}_{1, k}\right)^{-1}\left[\begin{array}{ll}
-I & I
\end{array}\right]^{T} G_{k-\tau}^{T}+\left(D_{k-\tau}-D_{o k-\tau}\right) \\
& \text { - }\left[\begin{array}{ll}
0 & I
\end{array}\right]\left(\Sigma_{k}^{-1}-\alpha_{k} \tilde{E}_{1, k}^{T} \tilde{E}_{1, k}\right)^{-1}\left[\begin{array}{ll}
-I & I
\end{array}\right]^{T} G_{k-\tau}^{T} \\
& \text { - }\left[\begin{array}{ll}
-I & I
\end{array}\right]^{T} C_{k}^{T}+\left(D_{k-\tau}-K_{o k} G_{k-\tau}\right)\left[\begin{array}{ll}
I & -I
\end{array}\right]\left(\Sigma_{k}^{-1}\right. \\
& \left.-\alpha_{k} \tilde{E}_{1, k}^{T} \tilde{E}_{1, k}\right)^{-1}\left[\begin{array}{ll}
-I & I
\end{array}\right]^{T} G_{k-\tau}^{T}+\left(D_{k-\tau}-D_{o k-\tau}\right) \\
& \text { - }\left[\begin{array}{ll}
0 & I
\end{array}\right]\left(\Sigma_{k}^{-1}-\alpha_{k} \tilde{E}_{1, k}^{T} \tilde{E}_{1, k}\right)^{-1}\left[\begin{array}{ll}
-I & I
\end{array}\right]^{T} G_{k-\tau}^{T}
\end{aligned}
$$

Substituting (35), (44), (45), (46), (47) and (48) into (49), we have

$$
\begin{aligned}
& {\left[A_{k}-K_{o k} C_{k} \quad K_{o k} C_{k}-A_{o k}\right]\left(\Sigma_{k}^{-1}-\alpha_{k} \tilde{E}_{1, k}^{T} \tilde{E}_{1, k}\right)^{-1}} \\
& {\left[\begin{array}{ll}
-I & I
\end{array}\right]^{T} C_{k}^{T}+\left[D_{k-\tau}-K_{o k} G_{k-\tau} \quad K_{o k} G_{k-\tau}-D_{o k-\tau}\right]} \\
& \bullet\left(\Sigma_{k-\tau}^{-1}-\gamma_{k} \tilde{E}_{3, k}^{T} \tilde{E}_{3, k}\right)^{-1}\left[\begin{array}{ll}
-I & I
\end{array}\right]^{T} G_{k-\tau}^{T} \\
& =\left(A_{k}-K_{o k} C_{k}\right)\left(\Xi_{k}^{-1}-\alpha_{k} E_{1, k}^{T} E_{1, k}\right)^{-1}\left(-C_{k}^{T}\right) \\
& +\left(D_{k-\tau}-K_{o k} G_{k-\tau}\right)\left(\Xi_{k-\tau}^{-1}-\gamma_{k} E_{3, k}^{T} E_{3, k}\right)^{-1}\left(-G_{k-\tau}^{T}\right)
\end{aligned}
$$

Substituting (50) into (26), then the optimal filter parameter $K_{o k}$ can be determined as follow

$$
\begin{aligned}
K_{o k}= & {\left[(1+\lambda) A_{k}\left(\alpha_{k}^{-1} I-E_{1, k} \Xi_{k} E_{1, k}^{T}\right)^{-1} C_{k}^{T}\right.} \\
& +\left(1+\lambda^{-1}\right) D_{k-\tau}\left(\Xi_{k-\tau}^{-1}-\gamma_{k-\tau} E_{3, k}^{T} E_{3,}\right) \mathrm{G}_{k-\tau}^{T} \\
& \left.+(1+\lambda) H_{1, k} H_{2, k}^{T}+\left(1+\lambda^{-1}\right) H_{3, k} H_{4, k}^{T}\right] R_{1, k}^{-1}
\end{aligned}
$$

where

$$
\begin{aligned}
& R_{1, k}=\left((1+\lambda) C_{k}\left(\Xi_{k}^{-1}-\alpha_{k} E_{1, k}^{T} E_{1, k}\right)^{-1} C_{k}^{T}+\left(1+\lambda^{-1}\right) G_{k-\tau}\left(\Xi_{k}^{-1}-\gamma_{k} E_{3, k}^{T} E_{3, k}\right)^{-1} G_{k-\tau}^{T}\right. \\
& \left.+L_{k}\left(R_{k}^{-1}-\beta_{k} E_{2, k}^{T} E_{2, k}\right)^{-1} L_{k}^{T}+\left((1+\lambda) \alpha_{k}^{-1}+\beta_{k}^{-1}\right) H_{2, k} H_{2, k}^{T}+(1+\lambda) \gamma_{k}^{-1} H_{4, k} H_{4, k}^{T}\right)
\end{aligned}
$$

Thus theorem 2 has been proved.

In the case of systems (1) without uncertainties and state delay factors, that is $D_{k-\tau}=0, G_{k-\tau}=0, H_{1, k}=0, H_{2, k}=0, H_{3, k}=0, H_{4, k}=0, E_{1, k}=0, E_{2, k}=0$, $E_{3, k}=0$ and $F_{k}=0$. Then substituting these values into (17)-(21) shows that the proposed robust filter will be equivalent to conventional kalman filter and then the error covariance matrix $\Xi_{k}$ will be 


$$
\Xi_{k+1}=\bar{\Xi}_{k+1}-K_{o k} J_{k+1} K_{o k}^{T}
$$

where

$$
\begin{aligned}
& J_{k+1}=(1+\lambda) C_{k} \Xi_{k} C_{k}^{T}+L_{k} R_{k} L_{k}^{T} \\
& \bar{\Xi}_{k+1}=(1+\lambda) A_{k} \Xi_{k} A_{k}^{T}+B_{k} Q_{k} B_{k}^{T} .
\end{aligned}
$$

\section{Simulation Results}

Consider the following uncertainty discrete time-varying system with state-delay in state and output matrices.

$$
\begin{aligned}
& x_{k+1}=\left[\left[\begin{array}{cc}
0 & 0.5 \\
0.2 & 0.3
\end{array}\right]+\left[\begin{array}{c}
0.5 \\
1
\end{array}\right] F_{k}\left[\begin{array}{ll}
0.2 & 0.1
\end{array}\right] \mid x_{k}\right.
\end{aligned}
$$

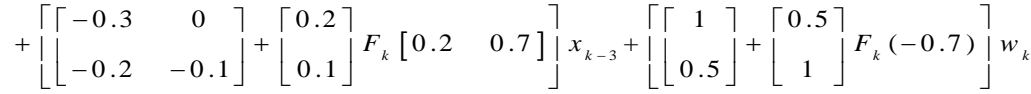

$$
\begin{aligned}
& y_{k}=\left[\left[\begin{array}{ll}
0.8 & 1
\end{array}\right]+4 F_{k}\left[\begin{array}{ll}
0.2 & 0.1
\end{array}\right]\right] x_{k} \\
& +\left[\left[\begin{array}{ll}
2 & 1
\end{array}\right]+2 F_{k}\left[\begin{array}{ll}
0.2 & 0.7
\end{array}\right]\right] x_{k-3}+\left[2.3+4 F_{k}(-0.7)\right] v_{k}
\end{aligned}
$$

where $F_{k}$ is a uncertainty matrix satisfying $F_{k} F_{k}{ }^{T} \leq I$, and $w_{k}$ and $v_{k}$ are mutually independent zero-mean Gaussian white noise sequences.

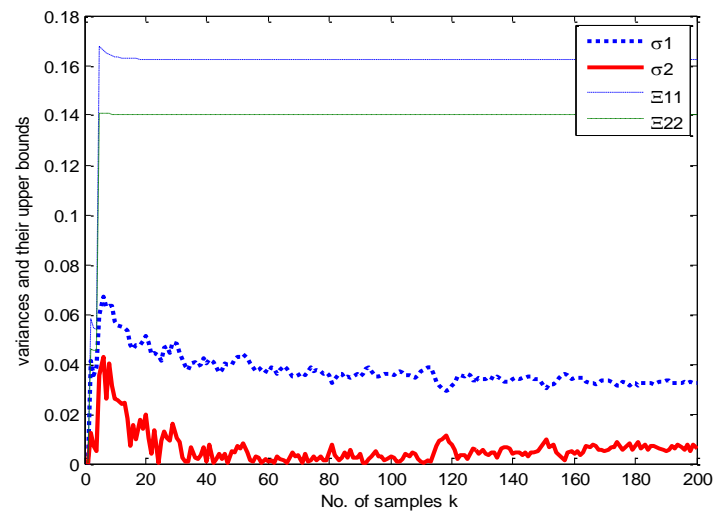

Figure 1. Upper Bounds $\Xi_{11}$ and $\Xi_{22}$ as well as Actual Variances $\sigma_{1}$ and $\sigma_{2}$ in the Case of $\alpha_{k}=5, \gamma_{k}=3, \beta_{k}=2$ and $\lambda=2.4$

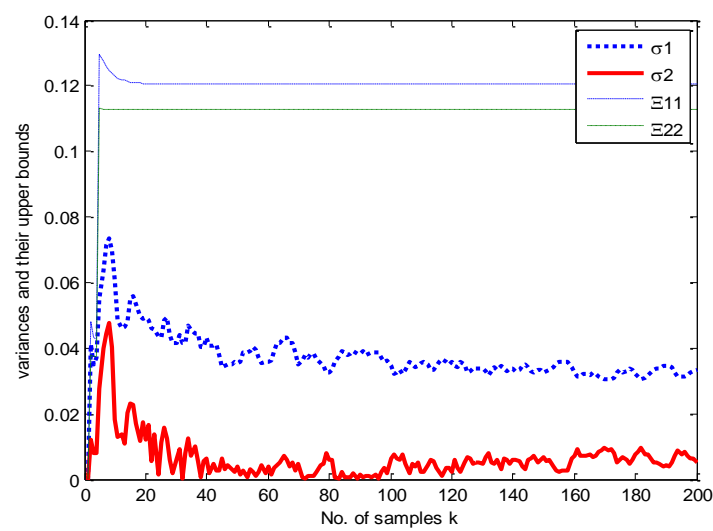

Figure 2. Upper Bounds $\Xi_{11}$ and $\Xi_{22}$ as well as actual variances $\sigma_{1}$ and

$\sigma_{2}$ in the Case of $\alpha_{k}=4, \gamma_{k}=3, \beta_{k}=2$ and $\lambda=2.4$ 
In the simulation, $F_{k}=\sin (6 k)$ and initial values are set as $x_{0}=\left[\begin{array}{ll}1 & 0\end{array}\right]^{T}$ and $\Xi_{0}=0.4 I$. The simulation results are given in Figures $1-5$. The plots of $\Xi_{11}$ and $\Xi_{22}$ are the upper bound and the plots of $\sigma_{1}=\operatorname{cov}\left(x_{1}-\hat{x}_{1}\right)$ and $\sigma_{2}=\operatorname{cov}\left(x_{2}-\hat{x}_{2}\right)$ are the actual variance for the state. It can be seen from Figures 1-2 that the value of upper bounds decline as the value of scalar parameter $\alpha_{k}$ decrease, but there may not find a feasible solutions to (20) and (21) if $\alpha_{k}$ is too small. So a practicable way to choose $\alpha_{k}$ is that make it as small as possible ensuring that (20) and (21) have solutions. The same conclusion on the $\gamma_{k}, \beta_{k}$ and $\lambda$ can be concluded from Figures 1-3, Figures 1-4 and Figures 1-5. In addition, we compare the sensitive of parameters $\alpha_{k}, \gamma_{k}, \beta_{k}$ and $\lambda$ to the proposed algorithm in Figures 1-5. It is can be concluded that the proposed algorithm is the most sensitive to the parameter $\lambda$, is the least sensitive to the parameter $\beta_{k}$, and is more sensitive to the parameter $\alpha_{k}$ than the parameter $\gamma_{k}$.

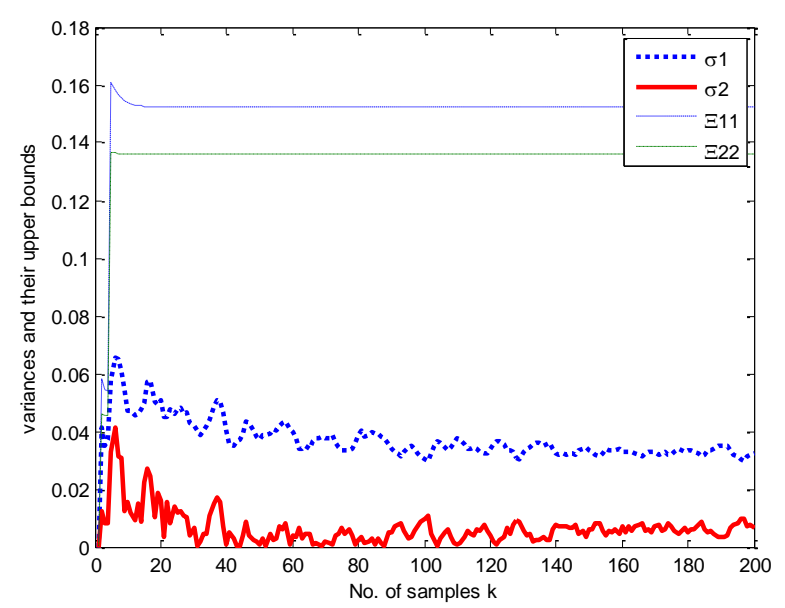

Figure 3. Upper Bounds $\Xi_{11}$ and $\Xi_{22}$ as well as Actual Variances $\sigma_{1}$ and $\sigma_{2}$ in the Case of $\alpha_{k}=5, \gamma_{k}=2, \beta_{k}=2$ and $\lambda=2.4$

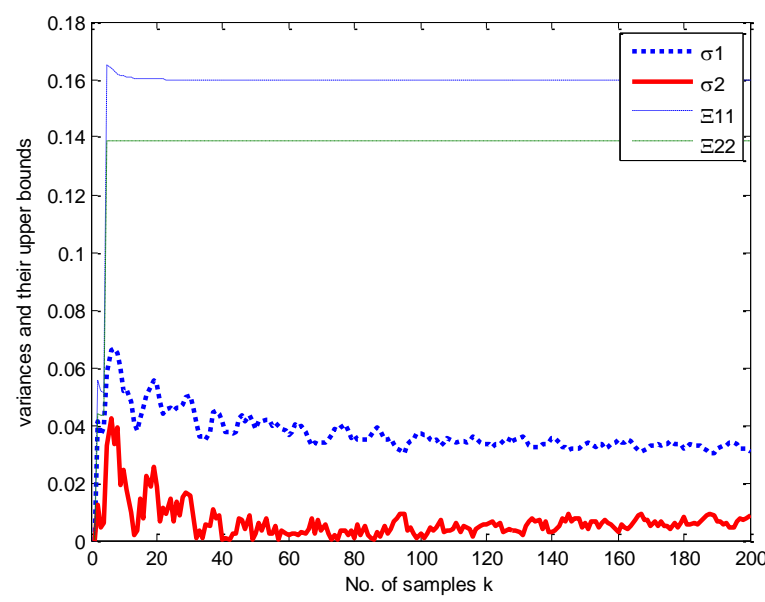

Figure 4. Upper Bounds $\Xi_{11}$ and $\Xi_{22}$ as well as Actual Variances $\sigma_{1}$ and

$\sigma_{2}$ in the Case of $\alpha_{k}=5, \gamma_{k}=3, \beta_{k}=1$ and $\lambda=2.4$ 


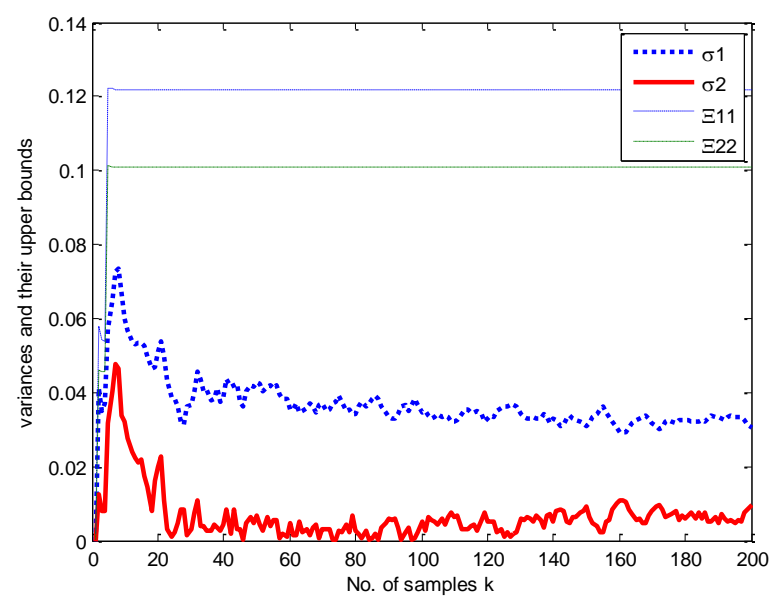
Figure 5. Upper Bounds $\Xi_{11}$ and $\Xi_{22}$ as well as Actual Variances ${ }^{\sigma_{1}}$ and$$
\sigma_{2} \text { in the Case of } \alpha_{k}=5, \gamma_{k}=3, \beta_{k}=2 \text { and } \lambda=1.4
$$

\section{Conclusion}

In this paper, a robust finite-horizon kalman filter for linear discrete time-varying system is proposed. The system under consideration is subject to constant delay both in state and output matrices and uncertainties in state matrix, the output process and the white noise covariance matrices. The existence of upper-bound on the estimation error covariance is proved. In addition, the upper bound on the estimation error covariance will be obtained without any uncertain parameters. The optimal filter parameters state, state delay and gain matrices are obtained by minimizing the upper-bound.

\section{References}

[1] B. D. O. Anderson and J. B. Moore, Optimal Filtering. Englewood Cliffs, NJ: Prentice-Hall, 1979.

[2] E. N. Goncalves, R. M. Palhares, and R. H. C. Takahashi, "filter design for systems with polytope-bounded uncertainty," IEEE Trans. Signal Processing, vol. 54, no. 9, pp. 3620-3626, Sep. 2006.

[3] U. Shaked and C. E. de Souza, "Robust minimum variance filtering," IEEE Trans. Signal Processing, vol. 43, pp. 2474-2483, Nov. 1995

[4] H. Gao and C. Wang, "Delay-dependent robust and filtering for a class of uncertain nonlinear time-delay systems,” IEEE Trans. Automat. Control, vol. 48, no. 9, pp. 1661-1666, Sep. 2003.

[5] Z. Duan, J. Zhang and C. Zhang, "Robust and filtering for uncertain linear systems," Automatica, vol, 42, pp. 1919-1926, 2006.

[6] H. Gao, X. Meng and T. Chen, "New Design of Robust H Filters for 2-D Systems," IEEE Trans. Signal Processing, vol. 15, pp. 217-220, 2008

[7] M. Fu, Carlos E. de Souza and Z. Luo, "Finite-Horizon Robust Kalman Filter Design," IEEE Trans. Signal Processing, vol. 49, no. 9, pp.2103-2112, sep. 2001

[8] X. Zhu, Y. C. Soh, and L. Xie, "Design and analysis of discrete-time robust Kalman filters," Automatica, vol. 38, pp. 1069-1077, 2002.

[9] F. Yang, Z. Wang and Y. S. Hung, "Robust kalman filtering for discrete time-varying uncertain systems with multiplicative noises," IEEE Trans. Automat. Control, vol. 47, no. 7, pp. 1179-1183, Jul. 2002.

[10] Z. Dong and Z. You, "Finite-horizon robust Kalman filtering for uncertain discrete time-varying systems with uncertain-covariance white noises," IEEE Signal Processing Lett, vol. 13, no. 8, pp. 493-496, Aug. 2006.

[11] M. S. Mahmoud, L. Xie and Y.C. Soh, "Robust Kalman filtering for discrete state-delay systems," IEE Proc.-Control Theory Appl., vol. 47.No. 6, pp. 613-618. Nov. 2000.

[12] L. Xie, Y. C. Soh and C. E. Souza, "Robust Kalman filtering for uncertain discrete-time systems," IEEE Trans. Automat. Control, vol. 39, no. 6, pp. 1310-1314, Jun. 1994.

[13] H. Henderson and S. Searle, "On deriving the inverse of a sum of matrices," SIAM Review, vol. 23, no. 1, pp. 53-60, Jan. 1981. 


\section{Authors}

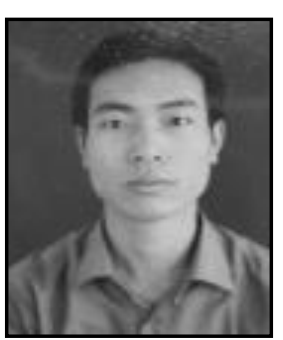

Jun-Hui Zheng. He received his M.Sc. in Information Sciences (2010) from University. Now he is lecturer of informatics at College of computer science and technology, PDS University. His current research interests include Artificial Intelligence and Control Techniques.

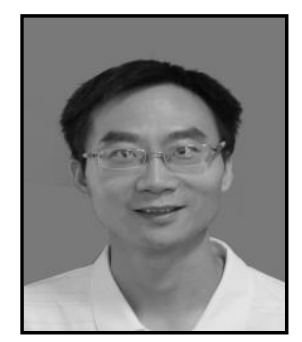

Quan Gan. He received his M.Sc. in Information Sciences (2008) from University. Now he is lecturer of informatics at College of computer science and technology, PDS University. His current research interests include Algorithm Analysis and Control Techniques. 